Jenina Rachel D. J. Escalderon, MD

Angelo A. Monroy, MD

Department of Otorhinolaryngology Head and Neck Surgery

University of Santo Tomas Hospital

\section{Kikuchi-Fujimoto Disease in a Filipino Boy: A Case Report}

\begin{abstract}
Objective: To report a case of a 12-year-old boy with Necrotizing Lymphadenitis (KikuchiFujimoto disease) presenting as lymphadenitis secondary to multiple diagnosis.
\end{abstract}

\section{Methods:}

\author{
Design: Case Report \\ Setting: Tertiary Private Hospital \\ Patient: One
}

Results: A 12-year-old boy consulted for two-month history of cervical lymphadenopathy with the underlying cause remaining unclear despite multiple consults, diagnosis and medical treatment. Lymphoma was considered and excision biopsy with further investigations confirmed a diagnosis of Kikuchi-Fujimoto disease. Supportive management was given with no recurrence of symptoms noted on 18 months of follow up.

Conclusion: Kikuchi-Fujimoto disease in this case was a diagnosis of exclusion. Even with a proper history and physical examination, experts in otolaryngology can be misled to manage this case as malignant. Awareness of the disease and appropriate examinations including immunohistochemistry are important for a timely diagnosis and proper intervention.

\section{Keywords: lymphadenitis; Kikuchi-Fujimoto disease; lymphoma; cervical lymph nodes}

First described in 1972 by Kikuchi and Fujimoto, Kikuchi-Fujimoto disease (KFD) is a condition seen more commonly in the East Asian and Japanese population with a slight female predominance (1.1 to $2.75: 1) .{ }^{1}$ Patients usually present with general flu-like symptoms and enlarged lymph nodes found at levels II to $\mathrm{V}$ of the neck. ${ }^{1-4}$ An estimated $30 \%$ of patients with KFD have been initially diagnosed and managed as malignant lymphoma subjecting the patient to unnecessary courses of chemotherapy. ${ }^{5}$

We present a case of a 12-year-old boy with a two-month history of lymphadenopathies and multiple consults and treatments. We aim to contribute awareness of this disease and pattern of condition that may lessen misdiagnosis, unnecessary interventions and inappropriate management. 


\section{CASE REPORTS}

\section{CASE REPORT}

A 12-year-old Filipino boy presented with a four-week history of a tender, firm swelling the size of rice grain on the left side of the neck and a spiking fever up to $39^{\circ} \mathrm{C}$. No other symptoms such as cough, colds, night sweats, weight loss or anorexia were reported. No other family members or close contacts were affected, there were no household pets and no history of recent travel. The initial diagnosis was "reactive lymphadenitis" thought to be secondary to an acute viral illness but Clindamycin was given for 1 week with no relief of symptoms.

Six weeks into the illness the patient's family consulted a pediatrician who confirmed enlarging tender lymphadenopathy with high grade fever of up to $40^{\circ} \mathrm{C}$. Several pruritic maculopapular lesions were noted that appeared to start from the chest, back or upper arms. A clinical diagnosis of mumps was made and the patient was advised to increase oral fluid intake. No medications were prescribed. No relief of symptoms was noted.

Seven weeks after the illness started symptoms were still present and a second pediatrician diagnosed both mumps and measles. Observation and supportive management of symptoms was advised. The maculopapular rash resolved spontaneously but the highgrade fever and mass on the left side of the neck left now measuring approximately $2 \times 3 \times 2 \mathrm{~cm}$ persisted.

Persistent symptoms 8 weeks into the illness led to an otorhinolaryngology referral with concerns of tuberculous adenitis. Results were negative for sputum acid-fast bacilli (AFB) and chest x-ray and purified protein derivative (PPD) tests were normal. The leukocyte count of 9.39 was also normal (normal value: $4-10.5 \times 10^{9} / L$ ) with a normal blood film.

A computed tomography (CT) scan of the neck (Figure 1) was requested with concerns that the patient had a lymphoma. Results revealed enlarged lymph nodes along the internal jugular chain with the largest at level I, measuring $1.9 \times 3.4 \times 2.7 \mathrm{~cm}$. There was subcutaneous fatty infiltration peripherally. Medially, the mass still exhibited a small fat plane between the mass and the deep soft tissues of the neck. These characteristics were non-definitive, and an excision biopsy was performed on the largest palpable level I lymph node, revealing atypical lymphoid proliferation. (Figure 2)

Immunohistochemical staining revealed negative results for S-100, CD30, CD3, CD20, CD15 and yielded positive results for CD 68. (Figure 3) These results were consistent with necrotizing lymphadenitis (KikuchiFujimoto disease).

Over the following months on regular follow-up, the patient was noted to have spontaneous resolution of symptoms. After 18 months' follow-up, he was apparently healthy with no recurrence of fever, rashes or tender lymphadenitis.

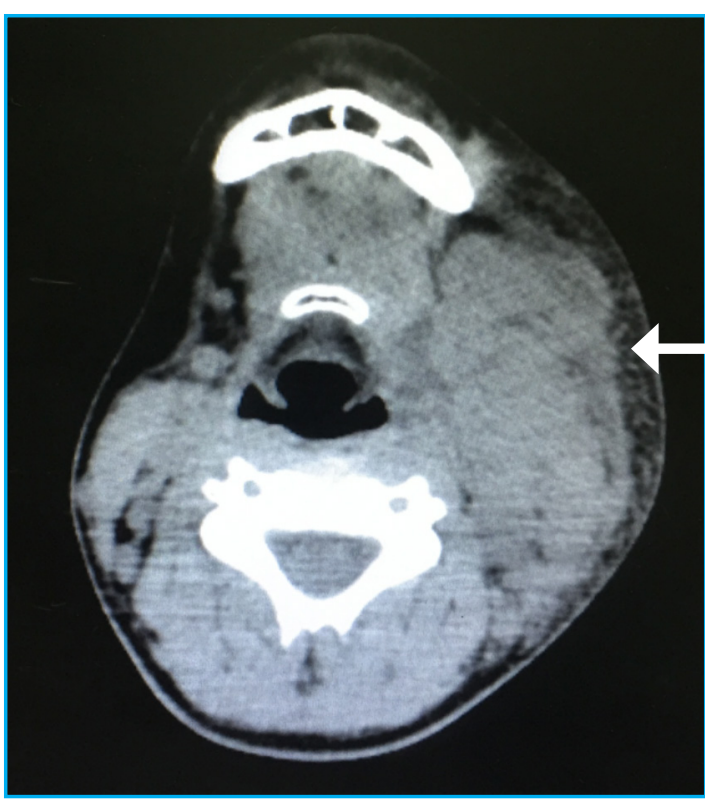

Figure 1. Non-contrast enhanced axial CT scan of neck at the level of the hyoid showing multiple confluent lymph nodes, left. Arrow: Level I confluent lymph nodes with necrotic areas.
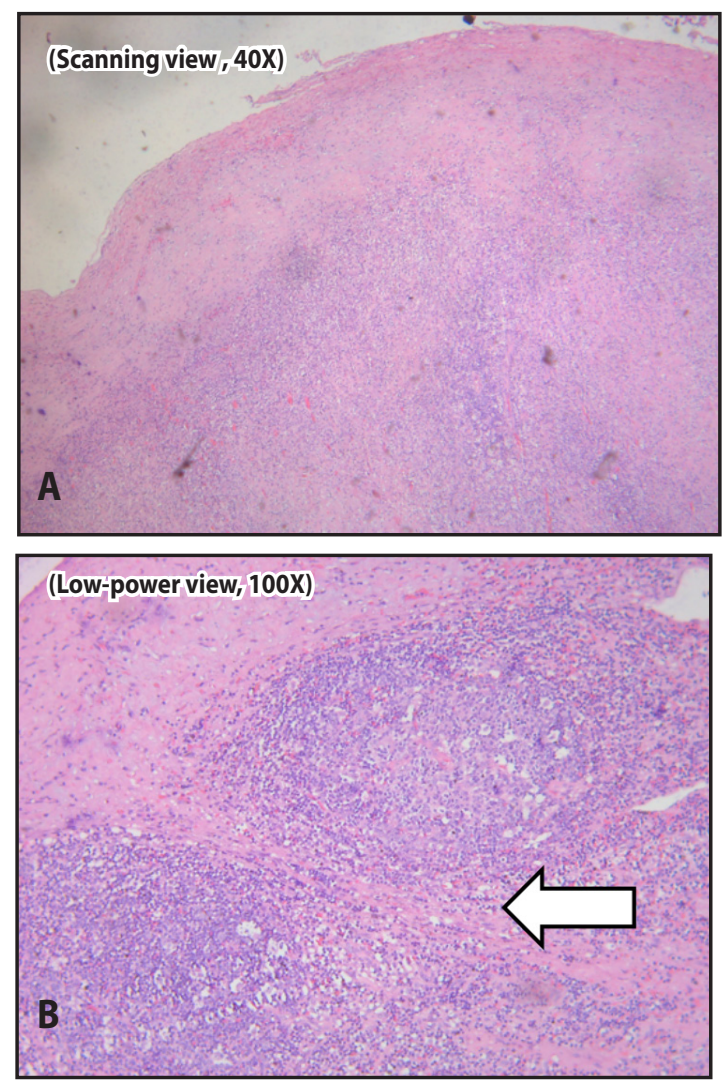

Figure 2. Histopathologic images, hematoxylin-eosin A. scanning view (40X) revealing a pathologic lymph node with no germinal center; and B. low-power view (100X) showing atypical lymphocytes with areas of necrosis containing acellular areas. (arrow) 


\section{CASE REPORTS}
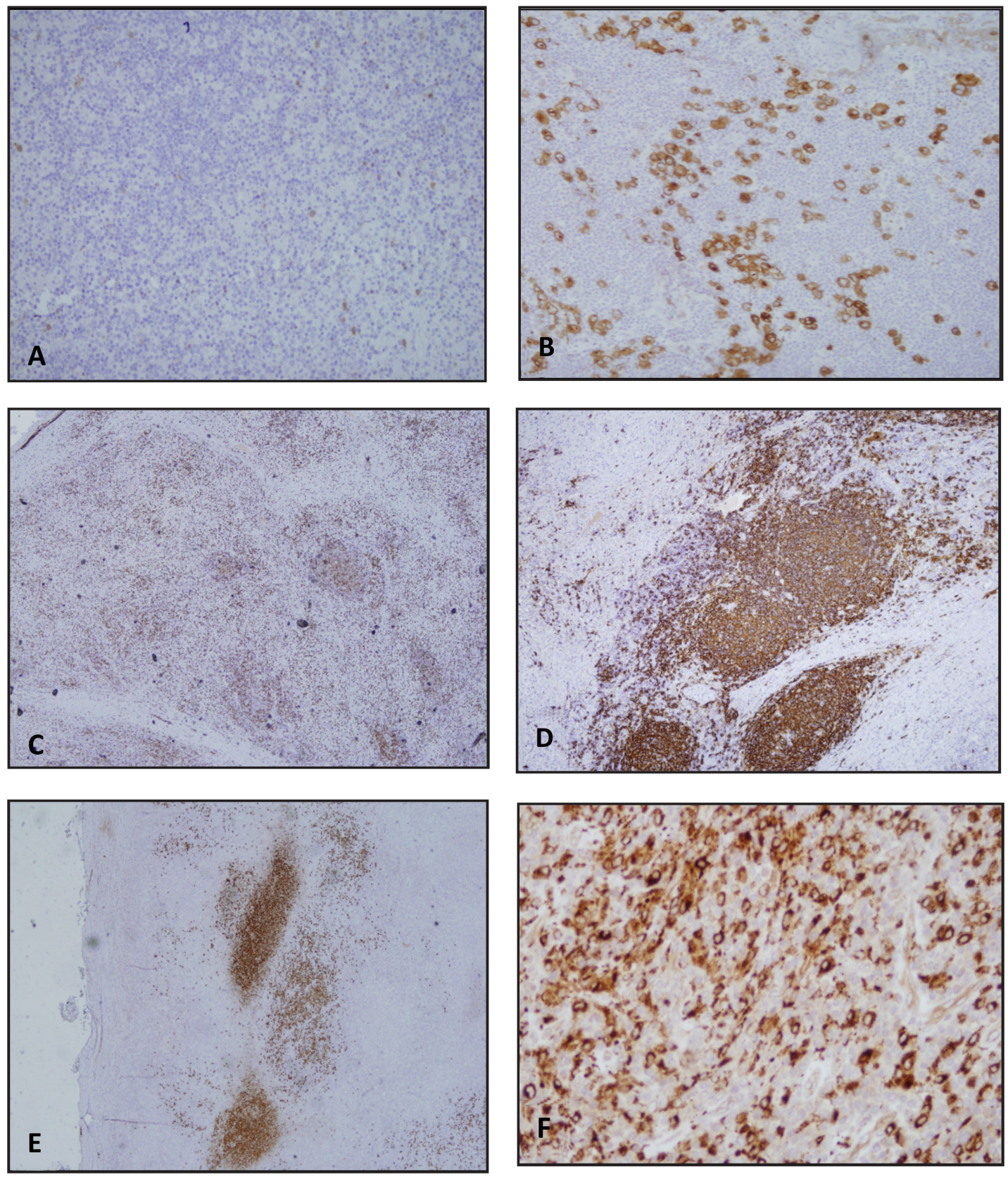

Figure 3. A-F Histopathologic with immunohistochemical staining images A. S100, B. CD30, C. CD3, D. CD20, E. CD15; and F. CD68

\section{DISCUSSION}

Kikuchi-Fujimoto Disease (KFD), also known as necrotizing histiocytic lymphadenitis is a rare benign condition involving the lymph nodes..$^{1-5}$ It is characterized by unilateral tender cervical lymphadenopathy mostly affecting the jugular carotid chain and posterior triangle that may be accompanied by a wide spectrum of systemic symptoms. ${ }^{2-5}$ Although it is likely to be underreported, to our best knowledge this is the first documented case of KFD in the Philippines.

Our case did not present in the typical age and gender group for this disease, as it is more common among young adult females, and the diagnosis was not considered until histology was reviewed. Initial investigations were centered on the most common etiologies of lymphadenopathy. Routine investigations contribute little to the underlying diagnosis of KFD. Approximately $25 \%$ to $31 \%$ of patients 


\section{CASE REPORTS}

with KFD are found to have atypical lymphocytes in their peripheral blood smear. ${ }^{6}$ As the patient presented with solid lymph nodes and a long history, a neck CT scan was done instead of ultrasonography. Studies have also showed that ultrasonography has a low sensitivity for KFD.7 Typical CT features described in a study were multiple homogenous lymphadenopathy involving level II to V; smaller than $2.5 \mathrm{~mm}$; perinodal infiltration and necrosis. ${ }^{4}$ The lymph node size seen in CT scan differentiates it from lymphoma. In lymphoma, the lymph node should usually be more than $3 \mathrm{~mm}$ while in KFD they are less than $3 \mathrm{~mm} .{ }^{4}$ This imaging was not definitive in our case and an excision biopsy was warranted.

The excision biopsy histopathological picture revealed necrotic acellular area and lymphoid cells with only a limited number of histiocytes. This was consistent with the usual histological picture of lymph nodes showing perivascular and interstitial inflammatory cell populations consisting of a mixture of lymphoid cells, histiocytes and karyorrhectic debris. ${ }^{2}$

Immunohistochemical staining for CD 68 was positive which is consistent with KFD. ${ }^{6}$ A positive $\mathrm{CD} 68$ reveals presence of macrophage phagocytic activity exhibiting a high rate of apoptosis that is typically seen in KFD but not in lymphoma. ${ }^{5}$

The etiology of KFD has not yet been established, but a number of case reports point towards a reaction to an underlying infection by bacteria (such as Yersinia enterocolitica, Brucellosis, Bartonella henselae, Entamoeba histolytica, Mycobacterium szulgai, Toxoplasma gondii), viral infection (HHV6, HHV8, parvovirus B19) or an underlying autoimmune disorder (Lupus). ${ }^{2}$ An upper respiratory prodrome, atypical lymphocytosis and lack of response to antibiotic therapy in affected patients are suggestive of a role of viruses in the pathogenesis of KFD. ${ }^{3}$ Further research is required to establish the precise cause/s.

As KFD is self-limiting and typically resolves within 1-4 months, the management is purely supportive. ${ }^{8}$ Analgesia, anti-inflammatory medications, nonsteroidal anti-inflammatory drugs and systemic corticosteroids have been used to alleviate the symptoms and to control fever and nodal tenderness. ${ }^{6}$ Corticosteroids have been recommended in cases of extra-nodal or generalized KFD for symptomatic control. Furthermore, definitive treatment for very large lymph nodes may be indicated in patients with airway compromise. ${ }^{9}$

Though recurrent cases (3.3\%) have been reported, ${ }^{4}$ it has a good prognosis with a mortality rate of only $2.1 \%$ among 244 cases. $^{10}$ Supportive therapy and close monitoring are important, as disseminated intravascular coagulopathy in conjunction with KFD can prove fatal. ${ }^{11}$

In conclusion, our case shows that the recognition of KFD in a patient can be problematic and may rely on exclusion of other causes such as lymphoma. Awareness of KFD and appropriate diagnostic evaluations that include immunohistochemical staining are important, because even experts in otolaryngology can be misled to manage such a case as malignant despite a proper history and physical examination.

\section{REFERENCES}

1. Baenas DF, Diehl FA, Haye Salinas MJ, Riva V, Diller A, Lemos PA. Kikuchi-Fujimoto disease and systemic lupus erythematosus. Int Med Case Rep J. 2016 Jun 29;9:163-7. doi: 10.2147/IMCRJ. S106396. PMID: 27418858; PMCID: PMC4935008.

2. Moinet $F$, Molinié V, Béraud G, Polomat $K$, Cordel N, Sainte-Marie $D$, et al. Epidemiology and Characteristics of Kikuchi-Fujimoto Disease in the African-Descent Population of Martinique, French West Indies. Arthritis Care Res (Hoboken). 2016 Dec;68(12):1883-1887. doi: 10.1002/ acr.22898. PMID: 27015112.

3. Bosch X, Guilabert A. Kikuchi-Fujimoto disease. Orphanet J Rare Dis. 2006 May 23;1(1):1-18. DOI: 10.1186/1750-1172-1-18; PMID: 16722618 PMCID: PMC1481509.

4. Kwon SY, Kim TK, Kim YS, Lee KY, Lee NJ, Seol HY. CT findings in Kikuchi disease: analysis of 96 cases. AJNR Am J Neuroradiol. 2004 Jun-Jul; 25(6): 1099-1102. PMID: 15205157.

5. Veer V, Lim A, Issing W. Kikuchi-Fujimoto disease: a case report and literature review. Case Rep Otolaryngol. 2012; 2012: 497604. DOI: 10.1155/2012/497604; PMID: 22953115 PMCID: PMC3420536.

6. Fiorella ML, Gelardi M, Marzullo A, Sabattini E, Fiorella R. Kikuchi-Fujimoto disease: an uncommon cause of neck swelling. Eur Arch Otorhinolaryngol. 2017 Mar: 274(3): 1761-1764. DOI: 10.1007/s00405-016-4147-6; PMID: 27317565.

7. Kim JY, Lee H, Yun B. Ultrasonographic findings of Kikuchi cervical lymphadenopathy in children. Ultrasonography. 2017 Jan;36(1);66-70. DOI: 10.14366/usg.16047; PMID: 27998053 PMCID: PMC5207361.

8. Veer V, Lim A, Issing W. Kikuchi-Fujimoto Disease: A Case Report and Literature Review. Case Reports in Otolaryngology 2012; 2012(Article ID 497604):5 pages. https://doi. org/10.1155/2012/497604.

9. Erhamamci, S, Reyhan M, Kocer NE. Kikuchi-Fujimoto disease as a rare cause of benign lymphadenopathy and (18) F-FDG PET/CT findings. Hell J Nucl Med. 2014 Jan-Apr; 17(1): 41-44. DOI: 10.1967/s002449910115; PMID: 24563878.

10. Kucukardali Y, Solmazgul E, Kunter E, Oncul O, Yildirim S, Kaplan M. Kikuchi-Fujimoto Disease: analysis of 244 cases. Clin Rheumatol. 2007 Jan; 26(1): 50-54. DOl: 10.1007/s10067-006-0230-5.

11. Barbat B, Jhaj R, Khurram D. Fatality in Kikuchi-Fujimoto disease: A rare phenomenon. World J Clin Cases. 2017 Feb 16; 5(2): 35-39. DOI: 10.12998/wjcc.v5.i2.35; PMID: 28255545 PMCID: PMC5314258. 